Conclusions EODLB is characterized by motor and neuropsychiatric symptoms while neuropsychological tests appear less reliable to differentiate EODLB from EOAD. Given that misdiagnosis of DLB remain significantly high, we propose a more careful and comprehensive clinical approach may improve the diagnosis rate.

Acknowledgement The NACC database is funded by NIA/NIH Grant U01 AG016976. NACC data are contributed by these NIA funded ADCs: P30 AG019610 (PI Eric Reiman, MD), P30 AG013846 (PI Neil Kowall, MD), P50 AG008702 (PI Scott Small, MD), P50 AG025688 (PI Allan Levey, MD, PhD), P50 AG047266 (PI Todd Golde, MD, PhD), P30 AG010133 (PI Andrew Saykin, PsyD), P50 AG005146 (PI Marilyn Albert, PhD), P50 AG005134 (PI Bradley Hyman, MD, PhD), P50 AG016574 (PI Ronald Petersen, MD, PhD), P50 AG005138 (PI Mary Sano, PhD), P30 AG008051 (PI Steven Ferris, PhD), P30 AG013854 (PI M. Marsel Mesulam, MD), P30 AG008017 (PI Jeffrey Kaye, MD), P30 AG010161 (PI David Bennett, MD), P50 AG047366 (PI Victor Henderson, MD, MS), P30 AG010129 (PI Charles DeCarli, MD), P50 AG016573 (PI Frank LaFerla, PhD), P50 AG016570 (PI Marie-Francoise Chesselet, MD, PhD), P50 AG005131 (PI Douglas Galasko, MD), P50 AG023501 (PI Bruce Miller, MD), P30 AG035982 (PI Russell Swerdlow, MD) , P30 AG028383 (PI Linda Van Eldik, PhD), P30 AG010124 (PI John Trojanowski, MD, PhD), P50 AG005133 (PI Oscar Lopez, MD), P50 AG005142 (PI Helena Chui, MD), P30 AG012300 (PI Roger Rosenberg, MD), P50 AG005136 (PI Thomas Montine, MD, PhD), P50 AG033514 (PI Sanjay Asthana, MD, FRCP), P50 AG005681 (PI John Morris, MD), and P50 AG047270 (PI Stephen Strittmatter, MD, PhD).

This study is supported by Singhealth Foundation Grant (NRS 15/001), NNI Centre Grant (NCG CS02) and National Medical Research Council, Singapore (NMRC/IRG/015).

\section{UNDERSTANDING FOREIGN ACCENT SYNDROME}

${ }^{1}$ Laura McWhirter ${ }^{*},{ }^{2}$ Nick Miller, ${ }^{3}$ Catriona Campbell, ${ }^{1}$ Ingrid Hoeritzauer, ${ }^{4}$ Andrew Lawton, ${ }^{1}$ Alan Carson, ${ }^{1} J$ Jon Stone. ${ }^{1}$ Centre for Clinical Brain Sciences, University of Edinburgh; ${ }^{2}$ Speech and Language Sciences, University of Newcastle upon Tyne; ${ }^{3}$ Department of Clinical Psychological Science, Maastricht University; ${ }^{4}$ Department of Psychological Medicine, Royal Infirmary of Edinburgh

\subsection{6/jnnp-2019-BNPA.21}

Objectives/Aims Foreign accent syndrome (FAS) is a disorder of speech in which listeners perceive the affected individual as speaking with a foreign or different regional accent that is not their habitual accent. FAS is widely understood as an unusual consequence of stroke or other lesions within speech-motor networks. However, case reports of FAS occurring in the absence of structural damage and difficulty identifying neural correlates has led to increasing recognition that that FAS sometimes represents a functional neurological disorder. We aimed to characterise symptoms, comorbidities, and features of recorded speech in individuals with selfreported FAS.

Methods Participants self-reporting FAS recruited from informal unmoderated online support forums and recruited via professional networks completed an online survey. Recorded samples of spontaneous speech and reading of a standardised text were analysed in a subgroup of 13 cases.

Results Forty-nine respondents (24 UK, 23 North America, 2 Australia) reported FAS of mean duration three years (range two months - 18 years). Common triggers were: migraine/ severe headache (15), stroke (12), surgery or injury to mouth or face (six), and seizure (five, including three non-epileptic). High levels of comorbidity included migraine (33), irritable bowel syndrome (17), functional neurological disorder (12), and chronic pain (12). Five reported structural lesions on imaging. Author consensus on aetiology divided into, 'probably functional' $(n=35,71 \%)$, 'possibly structural' $(n=4,8 \%)$, and 'probably structural'( $\mathrm{n}=10,20 \%)$, but positive features of functional FAS were present in all groups. Blinded analysis of speech recordings supplied by 13 respondents correctly categorised $11(85 \%)$ based on probable aetiology (functional vs structural) in agreement with case history assignment. Analysis of speech recordings identified a range of features demonstrating internal inconsistency with potential utility in the diagnosis of functional FAS.

Conclusions This study, of the largest FAS case series to date, details the experience and characteristics of individuals with self-reported FAS, and describes an approach to auditory-perceptual analysis of speech with potential diagnostic utility. Although conclusions are limited by the recruitment methods, high levels of functional comorbidity, symptom variability and additional linguistic and behavioural features suggest that chronic FAS may in many cases represent a functional neurological disorder, even when a structural lesion is present.

\section{MISSED DIAGNOSIS OF ADHD IN CHILDREN REFERRED TO A TIC DISORDER CLINIC}

Idura N Hisham*, Jeremy S Stern, Helen Simmons. St George's Hospital and St George's University of London

\subsection{6/jnnp-2019-BNPA.22}

Aim To examine whether Attention Deficit Hyperactive Disorder (ADHD), a common comorbid disorder in Tourette's Syndrome (TS) patients, is often missed by physicians referring to a Tic Disorder Clinic.

Method Referral letters and first clinic attendance reports for 119 new patients aged between 4-17 that attended a national tic disorder clinic between 2015-2017 were analysed to see how many new diagnoses of ADHD were made at first consultation that were not included in the referral letters. Other variables that were noted for each patient included age, sex, if referrer had a suspicion of ADHD (rather than established or firm diagnosis), medication for ADHD and the main treatment target decided at the tic disorder clinic.

Results Out of 119 patients 13 (11\%) already had a diagnosis of ADHD, which is in line with the prevalence of comorbid $\mathrm{ADHD}$ in the general population but not with the known increased prevalence in patients with TS (up to $80 \%$ in some studies). The assessment at the Tic Disorder Clinic found 46 cases of ADHD (38\%). Referrals were from pediatricians (51\%), general practitioners (35\%) and from mental health services $(10 \%)$.

Conclusions As the prevalence of comorbid ADHD is high in Tourette's patients and this can sometimes be obscured by the presentation of the tic disorder, referrers should have a low threshold for suspecting and managing ADHD in cases where specialist input for tics is awaited. It is likely that CAMHS referrals were under-represented in the sample and it may be expected that prior ADHD diagnoses would be more likely from that source. 